\title{
Imaging Criteria by Echocardiography for Detection of Hypertrophic Cardiomyopathy in Elite Athletes
}

\author{
Diana Hui Ping Foo, ${ }^{a}$ MD, Kai Huat Lam, ${ }^{c}$ MBBS, Mohamad Adam Bin Bujang, ${ }^{a}$ PhD, Andrew Kilung, ${ }^{\mathrm{b}}$ Dip, \\ Sally Suriani Ahip, ${ }^{d}$ MBBS, MMed, Tiong Kiam Ong, ${ }^{b}$ MBBS, Alan Yean Yip Fong, a, b MBChB \\ ${ }^{a}$ Clinical Research Centre, Sarawak General Hospital, Ministry of Health Malaysia, Kuching, Sarawak, 93586, Malaysia \\ ${ }^{b}$ Department of Cardiology, Sarawak Heart Centre, Ministry of Health Malaysia, Kota Samarahan, Sarawak, 94300, Malaysia \\ Assunta Heart Centre, Petaling Jaya, Selangor Darul Ehsan, 46990, Malaysia \\ Klinik Kesihatan Kota Sentosa, Ministry of Health Malaysia, Kuching Sarawak, 93250, Malaysia
}

Corresponding author: Diana Hui Ping Foo, MD,

Clinical Research Centre, Sarawak General Hospital, Jalan Hospital, 93586 Kuching, Sarawak, Malaysia

Mobile Phone number: +60168680633

Fax number: +6082276823

Email: dianafoo.crc@gmail.com

\begin{abstract}
BACKGROUND

Over the past few years, the number of sudden cardiac deaths in young elite athletes has been remarkable. Unfortunately, screening for hypertrophic cardiomyopathy $(\mathrm{HCM})$ is not mandatory in professional sports. Echocardiography remains the primary tool for assessing left ventricular (LV) structure and function. Therefore, we applied echocardiography to test whether echocardiography-derived variables accurately distinguish HCM from other variants of LV hypertrophy.
\end{abstract}

\section{METHODS}

A total of 82 subjects were enrolled and distributed into four groups, which comprised healthy controls $(\mathrm{HC})$, professional athletes, patients with hypertensive LV hypertrophy (HTN-LVH), and patients with HCM. All subjects were men. LV ejection fraction (LVEF) was preserved in all subjects. For each subject, 16 echocardiographic parameters of LV function and five parameters of LV structure were obtained and referenced against internationally recognized parameters. Speckle-tracking echocardiography was performed to obtain global longitudinal strain (GLS).

\section{RESULTS}

Elite athletes were found to have significantly $(p<0.05)$ lower LV mass index, septal wall thickness, relative wall thickness, and LV posterior wall thickness than HCM patients. Among patients with HTN-LVH, athletes also had significantly lower septal wall thickness. With respect to echocardiographic variables associated with LV function, elite athletes had significantly lower left atrial (LA) volume index, GLS, and septal tissue Doppler imaging (TDI) E/e' ratio and higher TDI e' velocity than $\mathrm{HCM}$ patients. Among HTN-LVH patients, TDI e' velocity was also significantly higher and TDI E/e' was significantly lower in elite athletes. On the receiver operating characteristic curve analysis, LV mass index $>122.2 \mathrm{~g} / \mathrm{m}^{2}$ (area under the curve [AUC] $=0.921$; $p<0.001)$, LA volume index $>34.73 \mathrm{ml} / \mathrm{m} 2(A \cup C=0.937 ; p<0.001)$, septal TDI E/e' ratio > 11.57 ( $A \cup C=0.866 ; p<0.001)$, and $G L S$ $<-15.35 \%(A \cup C=1.000 ; p<0.001)$ were variables distinguishing $\mathrm{HCM}$ among elite athletes.

\section{CONCLUSIONS}

LV mass index, LA volume index, TDI E/e' ratio, and GLS were found to be more strongly associated with $\mathrm{HCM}$. Therefore, we recommend that athletes with $\mathrm{LVH}$ in whom these echocardiographic variables exceed the cutoff values (as found in our population) should undergo comprehensive cardiovascular assessment before undertaking strenuous exercise.

\section{KEYWORDS}

Athlete's heart; Echocardiography; Hypertrophic cardiomyopathy; Global longitudinal strain; Left ventricular hypertrophy

\section{ABBREVIATIONS}

2D, two-dimensional; ANOVA, one-way analysis of variance; GLS, global longitudinal strain; HCM, hypertrophic cardiomyopathy; HTN, hypertension; LA, left atrial; LV, left ventricular; LVEF, left ventricular ejection fraction; LVH, left ventricular hypertrophy; $\mathrm{MRI}$, magnetic resonance imaging; ROC, receiver operating characteristic; TDI, tissue Doppler imaging

\section{INTRODUCTION}

Over the past few years, there have been a remarkable number of sudden cardiac deaths in elite athletes on the playing field. Among them, many involved professional soccer players who were young and physically fit. ${ }^{1}$ From a large registry in the United States of young, competitive athletes, majority of these sudden cardiac deaths were attributed to hypertrophic cardiomyopathy ( $\mathrm{HCM})^{2,3,4}$ However, screening for HCM is neither the current practice nor mandated in professional sports. ${ }^{5,6} \mathrm{HCM}$ may masquerade or be confused as athlete's heart, physiologic left ventricular (LV) adaptations due to increased hemodynamic load, neurohormonal changes as a result of intensive athletic training, ${ }^{7,8}$ and LV hypertrophy (LVH) secondary to hypertension (HTN). The true prevalence of HCM in Asian population is not well established due to scarcity of data from Asian countries compared to the Western counterparts. ${ }^{9,} 10$ Although echocardiography remains the primary tool for assessing LV structure and function, it is not properly utilized as routine pre-participation screening to characterize LV structural and functional adaptations in elite athletes in Asia. ${ }^{11}$ The echocardiography criteria and cutoff values for the detection of HCM were derived from data observed in Caucasian and Afro-American athlete populations in previous studies. ${ }^{12,13}$ Echocardiography's unparalleled accessibility, portability, lower cost, freedom from ionizing radiation and its capacity to characterize myocardial structure, systolic and diastolic function, and valve morphology and function with sufficient accuracy and detail ${ }^{14}$ merit its consideration as a first-line 
imaging modality to be included in pre-participation screening for most athletes. Thus, in this study, we employed echocardiography to test whether echocardiography-derived parameters related to LV structure and function accurately distinguish HCM from other variants of LV hypertrophy (LVH). This hypothesis was tested in four distinct groups, ie. healthy subjects as controls, professional athletes, patients with hypertensive LVH and patients with HCM.

\section{MATERIALS AND METHODS}

\section{STUDY POPULATION}

This cross-sectional case-control study was collaboration between clinical research center, cardiology referral center, and primary care setting locally in Sarawak region in Malaysia in 2015 to 2017. Subjects were screened for eligibility and enrolled into one of the following groups: healthy controls $(\mathrm{HC})$, professional athletes, patients with hypertensive LVH (HTN-LVH), and patients with $\mathrm{HCM}$. Healthy individuals were recruited from healthy volunteers screening program conducted by Sarawak General Hospital Clinical Research Centre; HCM patients who fulfilled the eligibility criteria were enrolled from cardiac specialist outpatient clinic at Sarawak Heart Centre; eligible hypertensive patients who attended primary care outpatient clinic follow up were enrolled from Klinik Kesihatan Kota Sentosa. Both Sarawak General Hospital Clinical Research Centre and Sarawak Heart Centre worked closely with the Sarawak State Football Association in 2015 and conducted a screening program for elite athletes.

The inclusion criteria for each study group were as follows:

(1) HC: Healthy individuals with no known medical illnesses, exhibiting sinus rhythm on 12-lead electrocardiogram and preserved LV ejection fraction (LVEF>55\%).

(2) HTN-LVH: Consecutive, asymptomatic, known hypertensive patients on at least 5 years of treatment, exhibiting LVH on 12lead electrocardiogram (following the Sokolow-Lyon criteria) and echocardiography (septal or posterior wall thickness $>1.1 \mathrm{~cm}$ ), preserved LVEF (LVEF>55\%), and no evidence of other underlying co-morbidity besides HTN.

(3) HCM: Consecutive, known patients with HCM diagnosed by cardiac magnetic resonance imaging (wall thickness $\geq 1.5 \mathrm{~cm}$ in one or more LV myocardial segments), ${ }^{15}$ with known history of implantable cardioverter-defibrillator (ICD) insertion, stroke, sudden cardiac arrest, preserved LVEF (LVEF>55\%), and presented with no evidence of other co-morbidity except HCM.

(4) All included professional athletes were highly trained elite footballers from the Sarawak State Football Association, and each had been competing at a national or international level consecutively in at least the last 5 years and engaged in similar, regimented, high-intensity endurance as well as isometric exercise training.

The exclusion criteria were as follows: subjects with significant valvular heart disease, prior myocardial infarction, known obstructive coronary artery disease, or heart failure. None of the $\mathrm{HC}, \mathrm{HTN}-\mathrm{LVH}$, and HCM subjects were engaged in competitive sports or systematic exercise training (i.e., <3 hours of exercise/ week).
The study protocol was approved by the Medical Research and Ethics Committee (MREC), Ministry of Health Malaysia (Research ID: NMRR-14-1051-22147). Written informed consent was obtained from all subjects involved in the study.

\section{ECHOCARDIOGRAPHY}

All subjects underwent standard echocardiographic examinations. All echocardiographic examinations were performed using Philips XMatrix iE33 machines (Philips Medical Systems, Andover, Massachusetts) equipped with S3 probes (2-4 MHz). All images acquired were digitally stored for offline analysis using Philips Xcelera version R3.1, level 1 workstation (Philips Medical Systems, Andover, Massachusetts).

All conventional echocardiography variables assessed in this study were associated with LV structure and LV function. Specifically, two-dimensional (2D) echocardiographic assessment of LV cavity diameters and septal and posterior wall thicknesses was performed according to European Association of Cardiovascular Imaging and American Society of Echocardiography criteria. ${ }^{16} \mathrm{LV}$ mass and relative wall thickness were calculated from these linear dimensions in a standard manner. Left atrial (LA) volume was calculated from the apical fourand two-chamber views using the modified Simpson's method and then indexed to the body surface area (BSA).

2D echocardiography variables of LV function that were assessed in this study included both LV systolic and diastolic functions. LVEF was calculated by the biplane Simpson's rule. To assess LV diastolic function, transmitral early $(E)$ and late $(A)$ pulsed wave Doppler diastolic peak flow velocities were measured in the apical four-chamber view, with the sample volume placed at the tip of the mitral leaflets. Tissue Doppler imaging (TDI) measurement of the mitral annular motion was achieved in the apical four-chamber view, with a 10-ml sample volume placed at the septal border of the mitral annulus. Early (e') and late (a') diastolic peak velocities and their ratio were recorded. ${ }^{12,17}$

In addition to conventional 2D echocardiography, 2D strain using ultrasound speckle tracking was utilized to characterize longitudinal systolic strain. Apical long, apical four- and twochamber views were acquired at an increased frame rate (50-70 frames/second). Cine loops of five cycles were digitally saved for offline analysis in a blinded manner, using a dedicated software package (QLab, Philips Medical System, Andover, Massachusetts). Using the Automatic Function Imaging feature of the software, a point-and-click approach was utilized to identify three anchor points (two basal and one apical), following which the software tracked the endocardial contour automatically. After initial tracing of the endocardial border and software processing, the operator confirmed an adequate tissue tracking. The LV was divided into 17 segments, and automated measurements of segmental systolic longitudinal strain values in the three apical views were then used to generate 17-segment polar map. Calculation of the mean longitudinal strain was derived as the LV global longitudinal strain in negative values. The higher negative values corresponded to higher strain (contractility). All images were analyzed offline by an experienced analyzer blinded to the diagnosis. For each echocardiographic variable, 3 readings were obtained out of 3 cardiac cycles and averaged. All readings were then validated by a second board-certified cardiologist with expertise in echocardiography. This cardiologist reviewed all the collected echocardiography data and images and was blinded to the clinical data of each subject. 


\section{STATISTICAL METHODS}

Baseline characteristics and echocardiography variables were presented as mean (standard deviation). Data distribution and normality were examined based on histogram, normality tests and values of skewness and kurtosis. No serious violations of normality assumptions were found. Hence, parametric test was applied in the statistical analysis. One-way analysis of variance (ANOVA) and post-hoc least significant difference tests were conducted to determine whether there was a significant difference in any parameters between the four groups. Receiver operating characteristic (ROC) curve analysis was conducted to determine the association between echocardiography parameters toward the state of HCM (HCM versus athletes; HCM versus HTN group). The optimal cutoffs were determined among the echocardiography parameters that are highly significant in discriminating $\mathrm{HCM}$ and non-HCM. All analyses were carried out using SPSS (IBM SPSS Statistics for Windows, Version 21.0. Armonk, NY)

\section{RESULTS}

A total of 82 subjects (mean age $39.2 \pm 15.3$, all men) were prospectively enrolled and distributed in four distinct study groups: 27 healthy controls (HC), 27 professional athletes, 14 patients with hypertensive LVH (HTN-LVH), and 14 patients with $\mathrm{HCM}$. Baseline characteristics are presented in Table 1. As might be expected, athletes were younger and taller and had lower diastolic blood pressure and heart rate compared to the other groups.

\section{ECHOCARDIOGRAPHY PROFILES}

The echocardiography profiles of the study population are depicted in Table 2. Of the conventional echocardiographic variables that were associated with LV structure, athletes showed similar LV cavity (end diastolic) but thinner septal and LV posterior wall thickness compared with patients with HCM; therefore, relative wall thickness (i.e., the ratio between LV posterior wall thickness and cavity size) was lower in athletes. The LV mass indexed to the BSA was lower; LA volume indexed to BSA was smaller in athletes than in patients with HCM. Septal wall thickness was also substantially thinner in athletes than in patients with HTN-LVH. On the contrary, athletes showed similar LV structure compared with sedentary HC.

Despite preserved LVEF, s' in the HCM cohort was significantly lower than that in athletes. Of pulsed Doppler-derived variables reported in Table 2, athletes showed similar E wave velocity but lower transmitral A-wave velocity compared with patients with HTN-LVH and patients with HCM; consequently, the E/A ratio was higher in athletes. With TDI, athletes showed the highest e' velocity than $\mathrm{HC}$, patients with $\mathrm{HTN}-\mathrm{LVH}$, and patients with $\mathrm{HCM}$; subsequently, the septal E/e' ratio (i.e., LV filling pressure) was the lowest in athletes compared with other study subgroups. HTN-LVH and HCM cohorts had lower e' velocity compared with athletes and $\mathrm{HC}$ cohorts with the lowest early diastolic velocities observed in the HCM cohort. Finally, the LV global longitudinal strain was the lowest in patients with $\mathrm{HCM}$ than in $\mathrm{HC}$, athletes, and patients with HTN-LVH. On the contrary, no differences in LV global longitudinal strain were identified among athletes, HC, and patients with HTN-LVH.

\section{DISCRIMINATING HCM FROM VARIANT FORMS OF LVH}

To assess the discriminatory ability of various echocardiography variables that were associated with LV structure and function, the ROC curve analysis was performed in separate study subgroups (Table 3). In the model with HCM and athletes, the LV global longitudinal strain, LV mass index, LA volume index, and TDI septal E/e' ratio showed better discriminatory abilities compared to other echocardiography variables. In this model, the LV global longitudinal strain had comparable discriminatory ability with the three other conventional echocardiography parameters, namely, LV mass index, LA volume index, and TDI septal E/e' ratio. In the model with HCM and HTN-LVH study subjects, the LV global longitudinal strain, LV mass index, and LA volume index showed better discriminatory abilities than other echocardiography variables. In this model, LV global longitudinal strain performed better than the conventional echocardiography parameters LV mass index and LA volume index that showed similar discriminatory ability.

\begin{tabular}{|c|c|c|c|c|c|}
\hline Demographics & $\begin{array}{c}\mathrm{HC} \\
\mathrm{n}=27\end{array}$ & $\begin{array}{c}\text { Athletes } \\
n=27\end{array}$ & $\begin{array}{c}\text { HTN-LVH } \\
n=14\end{array}$ & $\begin{array}{l}\mathrm{HCM} \\
\mathrm{n}=14\end{array}$ & p-value* \\
\hline Age & $41.7(10.7)$ & $25.9(4.7)$ & $55.1(16.1)$ & $47.6(13.9)$ & $b, c, d, e$ \\
\hline Height (cm) & $168.5(5.9)$ & $172.4(6.3)$ & $166.1(4.7)$ & $164.8(8.5)$ & $b, d, e$ \\
\hline Weight (kg) & $76.0(14.3)$ & $68.8(8.2)$ & $71.8(10.7)$ & $66.9(13.8)$ & $a, b$ \\
\hline Body surface area $\left(\mathrm{m}^{2}\right)$ & $2.8(0.3)$ & $3.0(0.2)$ & $2.8(0.2)$ & $2.1(0.6)$ & $a, b, e, f$ \\
\hline Systolic BP (mmHg) & $130.3(10.7)$ & $130.7(12.3)$ & $147.5(21.4)$ & $127.9(10.0)$ & $c, d, f$ \\
\hline Diastolic BP (mmHg) & $79.4(8.5)$ & $70.2(5.1)$ & $83.8(8.8)$ & $79.2(7.9)$ & $b, d, e$ \\
\hline Heart rate & $72.2(8.9)$ & $59.9(10.5)$ & $73.4(14.0)$ & $72.2(8.8)$ & $b, d, e$ \\
\hline
\end{tabular}

Data are presented as mean (SD).

${ }^{*} \mathrm{p}$-values were derived from one-way ANOVA test with LSD for post-hoc test. a=statistically significant $(p<0.05)$ difference between HC versus $\mathrm{HCM} ; \mathrm{b}=$ statistically significant $(\mathrm{p}<0.05)$ difference between $\mathrm{HC}$ versus athletes; $c=$ statistically significant $(p<0.05)$ difference between $\mathrm{HC}$ versus HTN-LVH; $d=$ statistically significant $(p<0.05)$ difference between athletes versus HTN-LVH; $e=$ statistically significant $(p<0.05)$ difference between athletes versus HCM; $f=$ statistically significant $(p<0.05)$ difference between HTN-LVH versus HCM. BP, blood pressure; HC, healthy controls; HCM, hypertrophic cardiomyopathy; HTN-LVH, hypertensive left ventricular hypertrophy 


\begin{tabular}{|c|c|c|c|c|c|}
\hline 2D echocardiography variables & $\begin{array}{c}\mathrm{HC} \\
\mathrm{n}=27\end{array}$ & $\begin{array}{c}\text { Athletes } \\
n=27\end{array}$ & $\begin{array}{c}\text { HTN-LVH } \\
n=14\end{array}$ & $\begin{array}{l}\mathrm{HCM} \\
\mathrm{n}=14\end{array}$ & p-value ${ }^{*}$ \\
\hline Septal wall thickness $(\mathrm{cm})$ & $1.0(0.2)$ & $1.1(0.2)$ & $1.3(0.3)$ & $2.0(0.7)$ & $a, c, d, e, f$ \\
\hline LV posterior wall thickness $(\mathrm{cm})$ & $0.9(0.2)$ & $1.0(0.2)$ & $1.0(0.3)$ & $1.4(0.4)$ & $a, e, f$ \\
\hline Relative wall thickness (RWT) & $0.4(0.1)$ & $0.4(0.1)$ & $0.5(0.2)$ & $0.7(0.2)$ & $a, e, f$ \\
\hline LV end-diastolic diameter (cm) & $4.8(0.4)$ & $5.0(0.5)$ & $5.0(1.2)$ & $4.2(0.6)$ & NS \\
\hline LV mass $(\mathrm{g})$ & $159.5(39.6)$ & $184.5(36.4)$ & $210.1(45.4)$ & $324.3(151.6)$ & $a, e, f$ \\
\hline LV mass indexed to BSA ( $\mathrm{g} / \mathrm{m} 2)$ & $84.8(16.9)$ & $101.7(19.6)$ & $115.7(26.6)$ & $186.6(91.8)$ & $a, c, e, f$ \\
\hline LA volume $(\mathrm{ml})$ & $41.9(13.0)$ & $50.7(11.8)$ & $58.0(17.4)$ & $89.4(35.7)$ & $a, c, e, f$ \\
\hline LA volume indexed to BSA (ml/m2) & $22.5(6.4)$ & $27.8(6.1)$ & $32.4(10.4)$ & $54.4(22.7)$ & $a, c, e, f$ \\
\hline $\operatorname{LVEF}(\%)$ & $63.0(6.7)$ & $61.5(6.2)$ & $66.3(6.0)$ & $66.5(12.1)$ & $\mathrm{d}, \mathrm{e}$ \\
\hline \multicolumn{6}{|l|}{ Transmitral peak velocities: } \\
\hline E wave $(\mathrm{cm} / \mathrm{s})$ & $77.4(15.4)$ & $87.0(15.1)$ & $76.8(21.9)$ & $75.7(23.9)$ & NS \\
\hline A wave $(\mathrm{cm} / \mathrm{s})$ & $65.4(15.4)$ & $47.5(13.4)$ & $78.1(21.5)$ & $67.0(25.5)$ & $b, c, d, e$ \\
\hline E/A ratio & $1.2(0.4)$ & $2.0(0.7)$ & $1.0(0.3)$ & $1.4(0.7)$ & $b, d, e$ \\
\hline \multicolumn{6}{|l|}{ Tissue Doppler imaging: } \\
\hline e' wave $(\mathrm{cm} / \mathrm{s})$ & $8.9(2.4)$ & $11.4(2.2)$ & $6.5(2.1)$ & $5.0(1.5)$ & $a, b, c, d, \epsilon$ \\
\hline a' wave $(\mathrm{cm} / \mathrm{s})$ & $9.2(1.5)$ & $7.1(1.7)$ & $9.5(2.0)$ & $7.2(2.7)$ & $a, b, d, f$ \\
\hline $\mathrm{s}^{\prime}$ wave $(\mathrm{cm} / \mathrm{s})$ & $7.7(1.5)$ & $8.2(1.5)$ & $7.6(1.3)$ & $6.9(1.3)$ & $\mathrm{e}$ \\
\hline E/e' ratio & $9.1(3.9)$ & $7.8(1.6)$ & $12.2(4.3)$ & $16.5(6.1)$ & $\mathrm{a}, \mathrm{c}, \mathrm{d}, \mathrm{e}, \mathrm{f}$ \\
\hline e'/a' ratio & $1.4(2.2)$ & $1.9(1.3)$ & $0.7(0.4)$ & $0.8(0.4)$ & $d, e$ \\
\hline LV global longitudinal strain (\%) & $-20.5(2.3)$ & $-21.1(1.7)$ & $-21.2(2.9)$ & $-10.7(3.5)$ & $a, e, f$ \\
\hline
\end{tabular}

Data are presented as mean (SD).

* $p$-values were derived from one-way ANOVA test with LSD for post-hoc test. a=statistically significant $(p<0.05)$ difference between HC versus HCM; $b=$ statistically significant $(p<0.05)$ difference between $\mathrm{HC}$ versus athletes; $\mathrm{c}=$ statistically significant $(p<0.05)$ difference between $\mathrm{HC}$ versus HTN-LVH; $d=$ statistically significant $(p<0.05)$ difference between athletes versus HTN-LVH; $=$ =statistically significant $(p<0.05)$ difference between athletes versus HCM; $f=$ statistically significant $(p<0.05)$ difference between HTN-LVH versus HCM.

BSA, body surface area; HC, healthy controls; HCM, hypertrophic cardiomyopathy; HTN-LVH, hypertensive left ventricular hypertrophy; LA, left atrial; LV, left ventricular; LVEF, left ventricular ejection fraction; RWT, relative wall thickness

\begin{tabular}{|c|c|c|c|c|}
\hline & \multicolumn{2}{|c|}{ Between $\mathrm{HCM}$ and athletes } & \multicolumn{2}{|c|}{ Between HCM and HTN-LVH } \\
\hline & AUC $(95 \% \mathrm{Cl})$ & p-value & AUC $(95 \% \mathrm{Cl})$ & $\mathrm{p}$-value \\
\hline Septal wall thickness $(\mathrm{cm})$ & $0.917(0.000,1.000)$ & $<0.001$ & $0.824(0.639,1.000)$ & 0.006 \\
\hline LV posterior wall thickness $(\mathrm{cm})$ & $0.860(0.738,0.981)$ & $<0.001$ & $0.769(0.582,0.956)$ & 0.022 \\
\hline Relative wall thickness & $0.904(0.000,1.000)$ & $<0.001$ & $0.811(0.637,0.985)$ & 0.008 \\
\hline LV mass index (g/m2) & $0.910(0.000,1.000)$ & $<0.001$ & $0.840(0.660,1.000)$ & 0.004 \\
\hline LA volume index (ml/m2) & $0.938(0.000,1.000)$ & $<0.001$ & $0.849(0.699,1.000)$ & 0.003 \\
\hline \multicolumn{5}{|l|}{ Transmitral velocities: } \\
\hline A wave $(\mathrm{cm} / \mathrm{s})$ & $0.753(0.557,0.949)$ & 0.013 & $0.385(0.158,0.611)$ & NS \\
\hline E/A ratio & $0.194(0.027,0.362)$ & 0.003 & $0.596(0.365,0.828)$ & NS \\
\hline \multicolumn{5}{|l|}{ Tissue Doppler imaging: } \\
\hline$e^{\prime}$ wave $(\mathrm{cm} / \mathrm{s})$ & $0.000(0.000,0.000)$ & $<0.001$ & $0.317(0.106,0.529)$ & NS \\
\hline a' wave $(\mathrm{cm} / \mathrm{s})$ & $0.486(0.256,0.716)$ & & $0.269(0.063,0.476)$ & NS \\
\hline $\mathrm{s}^{\prime}$ wave $(\mathrm{cm} / \mathrm{s})$ & $0.239(0.069,0.409)$ & 0.01 & $0.317(0.103,0.531)$ & NS \\
\hline E/e' ratio & $0.951(0.000,1.000)$ & $<0.001$ & $0.692(0.483,0.902)$ & NS \\
\hline e'/a' ratio & $0.088(0.000,0.212)$ & $<0.001$ & $0.529(0.297,0.761)$ & NS \\
\hline LV global longitudinal strain (\%) & $1.000(1.000,1.000)$ & $<0.001$ & $1.000(1.000,1.000)$ & $<0.001$ \\
\hline
\end{tabular}

AUC, area under the curve; HCM, hypertrophic cardiomyopathy; HTN-LVH, hypertensive left ventricular hypertrophy;

LA, left atrial; LV, left ventricular. 


\begin{tabular}{|c|c|c|c|c|c|}
\hline & AUC (95\% Cl) & Cutoff point & Sensitivity & Specificity & $\mathrm{p}$-value \\
\hline Global longitudinal strain (\%) & $1.000(1.000,1.000)$ & $<-15.4$ & $100 \%$ & $100 \%$ & $<0.001$ \\
\hline LA volume index (ml/m2) & $0.937(0.875,1.000)$ & $>34.7$ & $83.3 \%$ & $85.7 \%$ & $<0.001$ \\
\hline LV mass index (g/m2) & $0.921(0.828,1.000)$ & $>122.2$ & $83.3 \%$ & $90.5 \%$ & $<0.001$ \\
\hline E/e' ratio & $0.866(0.766,1.000)$ & $>11.6$ & $75.0 \%$ & $84.1 \%$ & $<0.001$ \\
\hline
\end{tabular}

AUC, area under the curve; LA, left atrial; LV, left ventricular

From the ROC curve analysis in these two models, four echocardiography variables were identified as the criteria to distinguish HCM from other forms of LVH in our cohort (Table 4). These four variables were LV global longitudinal strain, LA volume index, LV mass index, and septal TDI E/e' ratio. The most reliable cutoff for LV global longitudinal strain was achieved at the threshold value of $<-15.4 \%$ (sensitivity $100 \%$, specificity $100 \%$ ). Of the structural variables, LV mass index $>122.2 \mathrm{~g} / \mathrm{m}^{2}$ (sensitivity $83.3 \%$, specificity $90.5 \%$ ) and LA volume index $>34.7$ $\mathrm{ml} / \mathrm{m} 2$ (sensitivity $83.3 \%$, specificity $85.7 \%$ ) identified patients with HCM with high accuracy. Of the diastolic variables, TDI E/e' ratio $>11.6$ showed high sensitivity $(75.0 \%)$ and specificity (84.1\%).

\section{DIscussion}

This study identified four variables, i.e., LV global longitudinal strain, LA volume index, LV mass index and septal TDI E/e' ratio, as the echocardiographic criteria to distinguish HCM from other forms of $\mathrm{LVH}$.

LVH can occur as physiological adaptations to intense physical training (i.e., athlete's heart) or as pathological hypertrophy associated with HTN, HCM, and infiltrative disease. The LVH in athletes may overlap with pathological hypertrophy such as those seen in HCM or HTN-LVH. However, compared to HTN-LVH, LVH associated with HCM is a more worrisome cardiovascular disease that could be clinically silent but could predispose individuals to sudden deaths. Unfortunately, HCM is the single most common disease causally linked to athletic field deaths, accounting for about one-third of sudden deaths among young competitive athletes.2, 3, 4 Although certain strategies had been outlined to prevent sudden cardiac deaths in elite athletes, the utility of echocardiography in HCM screening is not mandated in competitive sports. ${ }^{6}$ Furthermore, the detection rate of HCM with history and physical examination screening in the athletic population appears to be relatively low. 3, 18, 19, 20

The echocardiography criteria and cutoff values described in previous studies for the detection of $\mathrm{HCM}$ were derived from Caucasians and African/American athlete populations and therefore might not be applicable in Asian athlete population, which is characterized by different patterns of LV remodeling. ${ }^{12,}$

${ }^{13}$ In the present analysis, we took advantage of studying distinct populations of highly trained athletes; untrained patients with LVH associated with HTN only; untrained patients with HCM whose diagnosis were supported by cardiac magnetic resonance imaging (MRI), who were symptomatic, and presented with pathological outcomes such as sudden cardiac arrests, stroke, requiring cardiopulmonary resuscitation (CPR) and ICU admission, or ICD implantations; and sedentary healthy individuals as controls, matched for sex.

\section{MORPHOLOGICAL COMPARISON BETWEEN STUDY POPULATION AND WESTERN POPULATION}

Similar to previous studies comparing elite athletes with sedentary patients with $\mathrm{HCM},{ }^{12}$ the athletes in our cohort showed homogenous distribution of wall thickness, with absolute differences of $0.1 \mathrm{~cm}$ between the thickest (septal wall) and thinnest (LV posterior wall) segments. These observations support the principle that physiologic LV remodeling is characterized by a symmetric increase in LV wall thickness as a consequence of the hemodynamic volume overload associated with exercise. ${ }^{7,8}$ In contrary, the asymmetric and heterogeneous patterns of LVH in HCM represent the phenotypic expression of genetic disease.

Previous studies reported LV cavity dimension as a good discriminator between athlete's heart and morphologically mild HCM. ${ }^{21,22, ~}{ }^{23}$ A recent report suggested LV cavity dimension (end diastolic) to be a particularly good discriminator, favoring physiological LVH over HCM with both sensitivity and specificity of $100 \% .{ }^{12}$ In our study cohort, however, no significant differences in LV cavity dimension were identified among athletes, patients with HTN-LVH, and patients with HCM.

In the ROC curve analysis for distinguishing $\mathrm{HCM}$ from other variants of $L V H$, rather than wall thickness, we found LV mass indexed to BSA to be a better discriminator between athlete's heart and HCM.

A recent report identified the cutoff value of $L A$ size according to the basic measurement of the LA anteroposterior diameter. ${ }^{12}$ Although very commonly used in routine clinical practice, the $\mathrm{LA}$ anteroposterior diameter is still inadequate for assessing morphologic changes in the LA associated with athletic conditioning..$^{20}$ Our study assessed and identified the cutoff value for LA size by measuring LA volume according to modified Simpson's biplane rule and indexing it to the BSA. An LA volume index of $34.7 \mathrm{ml} / \mathrm{m}^{2}$ had lower sensitivity compared to LA anteroposterior diameter as suggested in previous report, ${ }^{12}$ but the discriminatory ability was better with higher area under the curve, and the false-positive results were also lower.

\section{FUNCTIONAL COMPARISON WITH PREVIOUS STUDIES OF DOPPLER-DERIVED, TISSUE DOPPLER, AND 2D SPECKLE- TRACKING ECHOCARDIOGRAPHY PARAMETERS}

Among echocardiographic parameters of LV function, we found only two parameters, namely, LV global longitudinal strain and septal TDI E/e' ratio, to be better discriminators between athlete's heart and HCM compared to previous studies involving Caucasian and African/American populations. ${ }^{13}$

In accordance with previous literatures, ${ }^{24,25}$ we observed significantly lower LV global longitudinal strain in patients with $\mathrm{HCM}$ than in patients with HTN-LVH, athletes, and HC. Although Alfonso et al. ${ }^{24}$ noted mitral annular e' velocity to be superior to 
LV global longitudinal strain in differentiating LVH from HCM, our analysis, however, revealed that LV global longitudinal strain $<-15.4 \%$ favoring the diagnosis of HCM offers the best discriminatory ability among all other echocardiography parameters.

In addition, in contrast to previous reports, 12, 21, 24 the ROC curve analysis in our study did not suggest TDI mitral annular e' velocity to be a good discriminator. However, our study demonstrated higher area under the curve and better compromise between sensitivity and specificity when discriminating $\mathrm{HCM}$ from other variants of $\mathrm{LVH}$ with similar cutoff value identified for septal E/e' ratio. ${ }^{21}$

\section{CLINICAL IMPLICATIONS}

Sudden cardiac death in an apparently young and healthy athlete is a tragic event. Often, in Europe and America, tragic deaths involving elite athletes are highly publicized and generate considerable attention in the local community, which may commit significant financial resources to prevent future events. ${ }^{19}$ Over the years, experts in sports cardiology have worked to quantify the incidence of sudden cardiac deaths in athletes, identify risk factors, and develop pre-participation screening tools. Comparatively, in Asia, the incidence of sudden cardiac deaths in athletes has not been widely reported. The pre-participation screening strategies were adapted from recommendations by multiple societies based on the data which were derived from the European and American athletic populations. 5, 26, 27, 28, 29

Accurate identification of $\mathrm{HCM}$ in athletes has relevant clinical implications, because this disease is one of the most common causes of deaths on athletic field and usually represents the basis for disqualification from competitive and professional sports. 2, 23, 30, 31, 32 Diagnosis of HCM in young competitive athletes may be clinically challenging when the extent of LVH is mild and mimic athlete's heart. We recognized that cardiac MRI is the gold standard to confirm the diagnosis of $\mathrm{HCM}$; unfortunately, it is not made available easily, not to mention its associated cost. Hence, we need a cost-effective and easily available diagnostic tool like echocardiography for screening HCM. In this study, we have identified a few variables that make $\mathrm{HCM}$ significantly different from all other variants of $\mathrm{LVH}$. The cutoff values were identified for these variables using ROC curve analysis.

Our results have significant practical implications; to our knowledge, our study was the first of its kind in identifying echocardiographic criteria for detection of $\mathrm{HCM}$ in elite athletes in a South-East Asian population. Although not an absolute screening method and further investigations remain to be done with focus on Asian athletic populations, the echocardiographic screening using the four criteria is recommended to be the preparticipation evaluation tool prior to competitions in athletes.

\section{LIMITATIONS}

The authors recognized some limitations in this study. First, the relatively small number of athletes with $\mathrm{LVH}$, patients with HTN$\mathrm{LVH}$, and patients with $\mathrm{HCM}$ may underrepresent the overall spectrum of LVH. In view of the small sample size, only male patients were analyzed. A consistent finding across studies is that male athletes have a 3-5 times higher incidence of sudden cardiac deaths than female athletes. ${ }^{33,34}$ Second, the echocardiographic findings from our athlete cohort were derived from professional footballers only. It is worth comparing different patterns of LV remodeling among elite athletes from various sports disciplines, such as endurance athletes (e.g., runners, swimmers, cyclists), isometric sports athletes (e.g., weightlifters, bowlers), as well as other combined sports athletes (e.g., hockey players, basketball players, squash players) in future studies. In addition, the participants in all four study subgroups were not age-matched. The athletes in our study were much younger compared to patients with HCM and patients with HTN-LVH. Nevertheless, our study showed that the echocardiographic data were not confounded by age. The echocardiographic criteria described in our study had also demonstrated higher discriminatory ability with a significant cutoff value that distinguishes HCM from other variants of LVH. Finally, the cutoff values described in this study were derived from our local athlete population and therefore might not be directly applicable to other athlete populations, such as Caucasians, African Americans, or other Asians. ${ }^{13}$ In addition, our findings may not be extrapolated to patients with reduced LVEF.

\section{CONCLUSION}

Although future studies on Asian athletic population are warranted, LV mass index, LA volume index, septal TDI E/e' ratio, and LV global longitudinal strain were found to be the echocardiographic variables with higher accuracy to distinguish $\mathrm{HCM}$. Therefore, incorporation of echocardiography as imaging modality in pre-participation screening should be recommended to elite athletes before any competitive professional sporting events. We propose that athletes with LVH in whom these echocardiographic variables, i.e., LV mass index, LA volume index, septal TDI E/e' ratio and LV global longitudinal strain, exceed the cutoff values (as found in our population) should undergo comprehensive cardiovascular assessment before undertaking strenuous exercise. This practice would prevent sudden cardiac deaths of young athletes on a playing field.

\section{ACKNOWLEDGEMENTS}

We would like to thank the Director General of Health Malaysia for his permission to publish this article. We thank the State Football Association of Sarawak for their support in this study. We also acknowledge the support and contribution of nurses and doctors at Klinik Kesihatan Kota Sentosa.

\section{FUNDING}

This work was supported by research grant from the National Institute of Health, Ministry of Health of Malaysia [grant number NMRR-14-1051-22147]. The sponsor had no role in study design; in the collection, analysis and interpretation of data; in the writing of the report. The sponsor approved the article to be submitted for publication.

\section{CONFLICT OF INTEREST}

None.

\section{AUTHOR CONTRIBUTIONS}

Diana Hui Ping Foo Data curation, Funding acquisition, Investigation, Methodology, Project administration, Writing original draft

Kai Huat Lam Conceptualization, Investigation, Methodology, Supervision, Writing - review \& editing

Mohamad Adam Bin Bujang Formal analysis, Methodology, Validation 


\author{
Andrew Kilung Investigation, Methodology \\ Sally Suriani Ahip Resources \\ Tiong Kiam Ong Resources, Supervision \\ Alan Yean Yip Fong Conceptualization, Funding acquisition, \\ Resources, Supervision, Writing - review \& editing
}

All the authors have approved the final version of the manuscript.

\section{REFERENCES}

1. McGinn D. Why are high-performance athletes having heart attacks? The Globe and Mail. 2018 May 8; Available from: https://www.theglobeandmail. $\mathrm{com} /$ life/health-and-fitness/health/conditions/why-are-high-performanceathletes-having-heart-attacks/article4100522/

2. Maron, B.J., Doerer, J.J., Haas, T.S., Tierney, D.M., Mueller, F.O. Sudden deaths in young competitive athletes: analysis of 1866 deaths in the United States, 1980-2006. Circulation. 2009;119:1085-1092

3. Maron, B.J., Shirani, J., Poliac, L.C., Mathenge, R., Roberts, W.C., Mueller, F.O. Sudden death in young competitive athletes. Clinical, demographic, and pathological profiles. JAMA. 1996;276:199-204

4. Maron, B.J., Carney, K.P., Lever, H.M., Lewis, J.F., Barac, I., Casey, S.A. et al, Relationship of race to sudden cardiac death in competitive athletes with hypertrophic cardiomyopathy. J Am Coll Cardiol. 2003;41(6):974-980

5. Maron, B.J., Thompson, P.D., Ackerman, M.J., Balady, G., Berger, S. et al, Recommendations and considerations related to preparticipation screening for cardiovascular abnormalities in competitive athletes: 2007 update; a scientific statement from the American Heart Association Council on Nutrition, Physical Activity, and Metabolism: endorsed by the American College of Cardiology Foundation. Circulation. 2007;115:1643-1655

6. Dvorak, J., Kramer, E.B. Schmied, C.M., Drezner, J.A., Zideman, D. Patricios, J. et al, The FIFA medical emergency bag and FIFA 11 steps to prevent sudden cardiac death: setting a global standard and promoting consistent football field emergency care. Br J Sports Med. 2013;47:1199_ 1202

7. Pelliccia, A., Mar Pelliccia, A., Maron, B.J., Spataro, A., Proschan, M.A Spirito, P. The upper limit of physiologic cardiac hypertrophy in highly trained elite athletes. N Engl J Med. 1991;324:295-301

8. Sharma, S., Maron, B.J., Whyte, G., Firoozi, S., Elliott, P.M., McKenna, W.J. Physiologic limits of left ventricular hypertrophy in elite junior athletes: Relevance to differential diagnosis of athlete's heart and hypertrophic cardiomyopathy. J Am Coll Cardiol. 2002;40:1431-1436

9. Chan, N.Y. Sudden cardiac death in Asia and China. Are we different? J Am Coll Cardiol. 2016;67:590-592

10. Maron, B.J., Kalra, A. Hypertrophic cardiomyopathy in the developing world: focus on India. Eur Heart J. 2014;35:2492-2495

11. Keh, Y.S., Tan, P.J., Chai, S.C., Tan, B., Tong, K.L. Physiologic limits of cardiac remodelling in Asian competitive athletes - a single centre study. Ann Acad Med Singapore. 2018;47:230-232

12. Caselli, S., Maron, M.S., Urbano-Moral, J.A., Pandian, N.G., Maron, B.J., Pelliccia, A. Differentiating left ventricular hypertrophy in athletes from that in patients with hypertrophic cardiomyopathy. Am J Cardiol. 2014:114:1383-1389

13. Papadakis, M., Carre, F., Kervio, G., Rawlins, J., Panoulas, V.F., Chandra, N. et al, The prevalence, distribution, and clinical outcomes of electrocardiographic repolarization patterns in male athletes of African/ Afro-Caribbean origin. Eur Heart J. 2011;32:2304-2313

14. Baggish AL, Battle RW, Beaver TA, Border WL, Douglas PS, Kramer CM, et al. Recommendations on the use of multimodality cardiovascular imaging in young adult competitive athletes: A report from the American Society of Echocardiography in collaboration with the Society of Cardiovascular Computed Tomography and the Society for Cardiovascular Magnetic Resonance. J Am Soc Echocardiogr. 2020;33:523-549.

15. Authors/Task Force members, Elliott, P.M., Anastasakis, A., Borger, M.A., Borggrefe, M., Cecchi, F., Charron, P. et al, 2014 ESC guidelines on diagnosis and management of hypertrophic cardiomyopathy: the Task Force for the diagnosis and management of hypertrophic cardiomyopathy of the European Society of Cardiology (ESC). Eur Heart J. 2014;35:27332779
16. Lang, R.M., Badano, L.P., Mor-Avi, V., Afilalo, J., Armstrong, A., Ernande, L. et al, Recommendations for cardiac chamber quantification by echocardiography in adults: an update from the American Society of Echocardiography and the European Association of Cardiovascular Imaging. J Am Soc Echocardiogr. 2015:28:1-39.e14

17. Nagueh, S.F., Smiseth, O.A., Appleton, C.P., Byrd, B.F. 3rd, Dokainish $\mathrm{H}$., Edvardsen, $\mathrm{T}$. et al, Recommendations for the evaluation of left ventricular diastolic function by echocardiography: an update from the American Society of Echocardiography and the European Association of Cardiovascular Imaging. J Am Soc Echocardiogr. 2016;29:277-314

18. Maron, B.J., Pelliccia, A. The heart of trained athletes: cardiac remodeling and the risks of sports, including sudden death. Circulation. 2006;114:1633-1644

19. Emery, M.S., Kovacs, R.J. Sudden cardiac death in athletes. JACC Heart Fail. 2018;6:30-40

20. Harmon, K.G., Zigman, M., Drezner, J.A. The effectiveness of screening history, physical exam, and ECG to detect potentially lethal cardiac disorders in athletes: a systematic review/meta-analysis. J Electrocardiol. 2015;48(3):329-338

21. Sheikh, N., Papadakis, M., Schnell, F., Panoulas, V., Malhotra, A., Wilson, $M$. et al, Clinical profile of athletes with hypertrophic cardiomyopathy. Circ Cardiovasc Imaging. 2015;8:e003454

22. Maron, B.J. Distinguishing hypertrophic cardiomyopathy from athlete's heart: a clinical problem of increasing magnitude and significance. Heart 2005;91:1380-1382

23. Maron, B.J., Pelliccia, A., Spirito, P. Cardiac disease in young trained athletes. Insights into methods for distinguishing athlete's heart from structural heart disease, with particular emphasis on hypertrophic cardiomyopathy. Circulation. 1995;91:1596-1601

24. Afonso, L., Kondur, A., Simegn, M., Niraj, A., Hari, P., Kaur, R. et al, Twodimensional strain profiles in patients with physiological and pathological hypertrophy and preserved left ventricular systolic function: a comparative analyses. BMJ Open. 2012;2:e001390

25. Richand, V., Lafitte, S., Reant, P., Serri, K., Lafitte, M., Brette, S. et al, An ultrasound speckle tracking (two-dimensional strain) analysis of myocardial deformation in professional soccer players compared with healthy subjects and hypertrophic cardiomyopathy. Am J Cardiol. 2007;100:128-132

26. Maron, B.J., Zipes, D.P., Kovacs, R.J. Eligibility and disqualification recommendations for competitive athletes with cardiovascular abnormalities: preamble, principles, and general considerations: a scientific statement from the American Heart Association and American College of Cardiology. Circulation. 2015;132:e256-e261

27. LaBotz, M., Bernhardt, D. Preparticipation physical evaluation. Adolesc Med State Art Rev. 2015;26(1):18-38

28. Drezner, J.A., O'Connor, F.G., Harmon, K.G., Fields, K.B., Asplund, C.A., Asif, I.M. et al, AMSSM position statement on cardiovascular preparticipation screening in athletes: current evidence, knowledge gaps, recommendations, and future directions. Br J Sports Med. 2017;51(3):153-167

29. Corrado, D., Pelliccia, A., Bjørnstad, H.H., Vanhees, L., Biffi, A., Borjesson, M. et al, Cardiovascular pre-participation screening of young competitive athletes for prevention of sudden death: proposal for a common European protocol. Consensus Statement of the Study Group of Sport Cardiology of the Working Group of Cardiac Rehabilitation and Exercise Physiology and the Working Group of Myocardial and Pericardial Diseases of the European Society of Cardiology. Eur Heart J. 2005;26:516-524

30. Maron, B.J. Sudden death in young athletes. N Engl J Med. 2003:349:1064-1075

31. Maron, B.J., Zipes, D.P. Introduction: eligibility recommendations for competitive athletes with cardiovascular abnormalities-general considerations. J Am Coll Cardiol. 2005:45:1318-1321

32. Pelliccia, A., Fagard, R., Bjørnstad, H.H., Anastassakis, A., Arbustini, E., Assanelli, D. et al, Recommendations for competitive sports participation in athletes with cardiovascular disease: a consensus document from the Study Group of Sports Cardiology of the Working Group of Cardiac Rehabilitation and Exercise Physiology and the Working Group of Myocardial and Pericardial Diseases of the European Society of Cardiology. Eur Heart J. 2005;26:1422-1445

33. Van Camp, S.P., Bloor, C.M., Mueller, F.O., Cantu, R.C., Olson, H.G. Nontraumatic sports death in high school and college athletes. Med Sci Sports Exerc. 1995:27:641-647

34. Corrado, D., Basso, C., Rizzoli, G., Schiavon, M., Thiene, G. Does sports activity enhance the risk of sudden death in adolescents and young adults? J Am Coll Cardiol. 2003;42:1959-1963 